\title{
SÍNDROME DE BURNOUT EM PROFISSIONAIS DA ÁREA DA SAÚDE ATUANTES EM DOIS MUNICÍPIOS DO INTERIOR DE MINAS GERAIS - BRASIL
}

\author{
BURNOUT SYNDROME IN HEALTH PROFESSIONALS ACTING \\ ON TWO MUNICIPALITIES OF MINAS GERAIS - BRAZIL
}

\author{
Daiany de Carvalho, ${ }^{1}$ Natália Silva, ${ }^{1}$ José Alexandre Bachur, ${ }^{1,2}$ Juliana Luzia França Mesquita, ${ }^{1}$ Aline do Carmo \\ França-Botelho ${ }^{1}$ \\ ${ }^{1}$ Uniaraxá/Araxá-MG'Brasil; ${ }^{2}$ Unifran, Franca-SP/Brasil.
}

Autor correspondente: Aline do Carmo França-Botelho

e-mail: alinecfb@terra.com.br

\section{EDITORES}

Thiago Gomes Heck

(Unijuí-Brasil)

Adriane Cristina Bernat Kolankiewicz

(Unijuí-Brasil)

\section{EDITORES DE ÁREA}

Educação \& Saúde

Eva Teresinha de Oliveira Boff

(Unijuí-Brasil)

Fisioterapia \& Saúde

Eliane Roseli Winkelmann

(Unijuí-Brasil)

Ciências Farmacêuticas \& Saúde

Marilei Uecker Pletsch

(Unijuí-Brasil)

Nutrição \& Saúde

Lígia Beatriz Bento Franz

(Unijuí-Brasil)

Nadia Oliveira

(Unipampa-Brasil)

Ingrid Perry

(UNESC-Brasil)

Enfermagem e suas contribuições para a prática

Adriane Cristina Kolankiewicz

(Unijuí-Brasil)

Crhis de Brum

(UFFS-Brasil)

Neila de Souza

(UFSM-Brasil)

\section{Exercício Físico \& Saúde}

Thiago Gomes Heck

(Unijuí-Brasil)

Anderson Zampier Ulbrich

Editora Unijuí

Universidade Regional do Noroeste do

Estado do Rio Grande do Sul (Unijuí)

\section{RESUMO}

Burnout é uma síndrome psicológica resultante de estressores interpessoais crônicos no trabalho e caracteriza-se por: exaustão emocional, despersonalização e diminuição do envolvimento pessoal no trabalho. Os profissionais de saúde têm sido destacados como muito vulneráveis ao Burnout, pois estão expostos diariamente a um ambiente de trabalho propício, lidam com doenças, sofrimento e morte. O objetivo do estudo foi verificar se há Burnout em profissionais da área da saúde atuantes em duas cidades do interior de Minas Gerais, Araxá e Santa Juliana. Trata-se de uma pesquisa de campo, de natureza quantitativa, exploratória, transversal, realizada com 75 profissionais. Foi utilizado o Inventário de Maslach para o Burnout - para Pesquisa em Serviços de Saúde (Maslach Burnout Inventory - Human Services Survey - MBI HSS). As médias globais da amostra foram: 20,2\%, 6,8\% e 34,7\%, para exaustão emocional, despersonalização e diminuição da realização pessoal, respectivamente. Esses percentuais são classificados como níveis médios de Burnout em todas as dimensões. Quanto à análise individual, observou-se um nível alto nas três dimensões simultaneamente em $6,7 \%$ (5 voluntários) da amostra. A presença de pelo menos uma dimensão em nível alto foi encontrada em $62,5 \%$ (47 voluntários). Os resultados indicam a presença de Burnout na amostra, o que pode comprometer a saúde e qualidade de vida, bem como, ter repercussões negativas no desempenho profissional.

Palavras-chave: Saúde do trabalhador. Estresse ocupacional. Burnout.

Submetido em: 9/5/2016

Aceito em: 2/7/2016 


\section{Revista}

\section{Contexto}

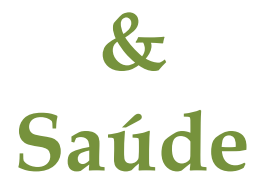

Volume 16

Número 31

2016

ISSN 2176-7114

A Revista Contexto \& Saúde é um periódico do Departamento de Ciências da Vida da Universidade Regional do Noroeste do Estado do Rio Grande do Sul (Unijuí). É um periódico semestral que tem por objetivo a divulgação da produção técnico-científica de temas relacionados à área de Ciências da Saúde.

O escopo da revista abrange a divulgação de resultados de pesquisa que contemplem avanços no processo saúde-doençacuidado e no conhecimento e aplicabilidade de novos processos químicos e biológicos em saúde.

Neste periódico, entende-se que a publicação de estudos com os aspectos epidemiológicos, assistenciais e educacionais em saúde, experimentais e aplicados é uma forma a subsidiar e qualificar a atenção à saúde de modo interdisciplinar.

\begin{abstract}
Burnout is a psychological syndrome resulting from chronic interpersonal stressors at work and is characterized by: emotional exhaustion, depersonalization and decrease Involvement Staff at Work. Health professionals have been highlighted as very vulnerable to burnout because they are exposed daily to an enabling work environment, dealing with illness, suffering and death. The aim of the study was to determine whether there is burnout in health care professionals working in two cities of Minas Gerais, Araxá and Santa Juliana. This is a field research, quantitative, exploratory, cross, performed with 75 professionals. Inventory Maslach Burnout was used for - for Health Research Services (Maslach Burnout Inventory Human Services Survey - MBI HSS). The overall sample averages were: 20.2\%, 6.8\% and $34.7 \%$ for emotional exhaustion, depersonalization, and reduced personal accomplishment, respectively. These percentages are classified as average levels of burnout in all dimensions. As for individual analysis, there was a high level in all three dimensions simultaneously in $6.7 \%$ (5 volunteers) of the sample. The presence of at least one dimension at a high level was found in $62.5 \%$ ( 47 volunteers). The results indicate the presence of Burnout in the sample, which may compromise the health and quality of life, as well as adversely affect the professional performance of these professionals.
\end{abstract}

Keywords: Occupational health. Occupational stress. Burnout. 


\section{INTRODUÇÃO}

O estresse ocupacional tornou-se uma importante fonte de preocupação e é reconhecido como um dos riscos mais sérios ao bem-estar psicossocial do indivíduo. O estresse relacionado ao trabalho coloca em risco a saúde dos membros das organizações e tem como consequências o desempenho ruim, baixo moral, alta rotatividade, absenteísmo e violência no local de trabalho (ROSSI; PERREWÉ; SAUTER, 2005).

Nas situações cotidianas do trabalho o profissional encontra-se constantemente vivenciando situações de tensão e estresse. As pessoas respondem a isso de maneiras diferentes, mas quando os níveis de ansiedade, tensão, competitividade e perfeccionismo são altos, o risco de estresse e Burnout são significativos (XAVIER; RIOS; FRANÇA-BOTELHO, 2013).

A síndrome é observada com mais frequência em pessoas competentes, dedicadas, comprometidas, responsáveis e motivadas que não conseguem suportar o grande estresse contido em seu trabalho, possuem grande desejo de ajudar os outros e preocupação social constante, mas não estão preparadas para as frustrações (MULATO, 2008).

Maslach, Schaufeli e Leiter (2001) afirmam que o Burnout é uma síndrome psicológica resultante de estressores interpessoais crônicos no trabalho e caracteriza-se por: exaustão emocional, despersonalização e diminuição do envolvimento pessoal no trabalho.

A exaustão emocional ocorre quando o indivíduo percebe não possuir mais condições de despender energia que o seu trabalho requer. Algumas das causas apontadas para a exaustão é a sobrecarga de atividades e o conflito pessoal nas relações, entre outras. A despersonalização, considerada uma dimensão típica da Síndrome de Burnout, é um elemento que a distingue do estresse, apresenta-se como uma maneira de o profissional se defender da carga emocional derivada do contato direto com o outro. Devido a isso, desencadeiam-se atitudes insensíveis em relação às pessoas nas funções que desempenha, ou seja, o individuo cria uma barreira para não permitir a influência dos problemas e sofrimentos alheios em sua vida. O profissional acaba agindo com cinismo, rigidez ou até mesmo ignorando os sentimentos dos outros. Já a diminuição da realização pessoal ocorre na sensação de insatisfação que a pessoa passa a ter com ela própria e com a execução de seus trabalhos, gerando sentimentos de incompetência e baixa autoestima (FRANÇA et al., 2014).

O profissional que tem a manifestação do Burnout ou está em processo de desenvolvimento apresenta exaustão física e mental, sente-se sem ânimo e expectativas para dar continuidade a seus projetos (CAMPOS, 2005).

De acordo com Benevides-Pereira (2008), os sintomas de Burnout podem ser subdivididos em físicos, psíquicos, comportamentais e defensivos. Entre os físicos destacam-se a fadiga constante e progressiva, os distúrbios do sono, as dores musculares e osteomusculares, as cefaleias, as enxaquecas, as perturbações gastrointestinais, as imunodeficiências, os transtornos cardiovasculares, etc. Quanto aos psíquicos são comuns a falta de atenção, as alterações de memória, a lentificação do pensamento, o sentimento de alienação e solidão, depressão e baixa autoestima. Os comportamentais envolvem a negligência ou excesso de escrúpulos, a irritabilidade, a agressividade, a incapacidade para relaxar, a dificuldade na aceitação de mudanças, a perda de iniciativa e aumento do consumo de substâncias como: bebidas alcoólicas, café, fumo, tranquilizantes, substâncias ilícitas, entre outras. Já os defensivos são: tendência ao isolamento, sentimento de onipotência, perda do interesse pelo trabalho ou até para o lazer, absenteísmo, ironia e cinismo. 
Segundo Carvalho e Malagris (2007), alguns fatores que contribuem para o aparecimento do estresse são: a instabilidade no trabalho, problemas financeiros, carga excessiva de atividades e pressões psicológicas. Alguns exemplos podem ser citados, como o aumento do volume de trabalho, conflitos diários no trabalho, falta de controle da situação, pressões, incompreensão dos chefes, ambiente desfavorável e função não adequada ao indivíduo.

Os profissionais de saúde têm sido destacados como muito vulneráveis ao Burnout. O contato muito próximo estabelecido com os pacientes pode mobilizar emoções e conflitos, tornando esses trabalhadores particularmente susceptíveis ao sofrimento psíquico, conduzindo a um adoecimento relacionado ao trabalho. Muitas vezes têm de lidar com dor, pacientes em estado grave e compartilhar da angústia deles e de seus familiares. Ao lidar com a frustração dos inevitáveis fracassos e com o sentimento de impotência quando a realidade se impõe, sentimentos de angústia, de dor e de medo são estressores potenciais (ARANDABELTRÁN et al., 2004; RIOS, 2008; DIAS, QUEIROS; CARLOTTO, 2010).

O objetivo do presente estudo foi verificar se ocorre Burnout em profissionais da área da saúde atuantes em Araxá ou em Santa Juliana, municípios do interior de Minas Gerais.

\section{METODOLOGIA}

Trata-se de uma pesquisa de campo, de natureza quantitativa, exploratória, transversal, realizada entre os meses de março a setembro de 2015. Trabalhou-se com amostra aleatória simples de 75 profissionais da área da saúde (equipe de enfermagem, fisioterapeutas, terapeutas ocupacionais, cirurgiões-dentistas, educadores físicos e psicólogos) atuantes em Araxá ou em Santa Juliana (MG).

Para avaliar a Síndrome de Burnout foi utilizado o Inventário de Maslach para o Burnout - para Pesquisa em Serviços de Saúde (Maslach Burnout Inventory - Human Services Survey - MBI HSS), autoaplicável, validado e traduzido para a língua portuguesa (BENEVIDES-PEREIRA, 2001).

O instrumento avalia as três dimensões da síndrome: a exaustão emocional, a despersonalização e a diminuição da realização pessoal. A exaustão emocional pretende analisar sentimentos de sobrecarga emocional e a incapacidade para dar resposta às exigências interpessoais do trabalho; é constituída por nove itens. A despersonalização busca medir "respostas frias", impessoais ou mesmo negativas, dirigidas para aqueles a quem prestam serviços. É constituída por cinco itens. A baixa realização pessoal é usada para avaliar sentimentos de incompetência e falta de realização e é constituída por oito itens. A forma de pontuação dos itens pesquisados adota a escala do tipo Likert que varia de zero a seis: (0) nunca, (1) uma vez ao ano ou menos, (2) uma vez ao mês ou menos, (3) algumas vezes no mês, (4) uma vez por semana, (5) algumas vezes por semana e (6) todos os dias.

A análise dos dados foi por estatística descritiva após os cálculos das pontuações do Burnout. A classificação para a exaustão emocional considera um nível de Burnout elevado quando são encontrados valores $\geq 27$ pontos; valores entre 19 e 26 pontos indicam níveis médios de Burnout e valores $\leq 18$ pontos correspondem a níveis de Burnout baixos. Quanto à despersonalização, pontuações $\geq 10$ pontos indiciam níveis altos de Burnout, valores entre 6 e 9 pontos apontam para níveis médios de Burnout e valores $\leq 5$ pontos indicam níveis baixos de Burnout. Por último, em relação à diminuição da realização pessoal, a sua leitura é oposta às anteriores, isto é, valores $\geq 40$ pontos indicam níveis baixos de Burnout, valores entre 34 e 39 pontos revelam níveis médios de Burnout e $\leq 33$ pontos indicam níveis altos de Burnout (SANTOS, 2009). 
A pesquisa seguiu todos os parâmetros da Resolução 466/2012 do Conselho Nacional de Saúde que regulamenta pesquisas com seres humanos. O trabalho foi avaliado e aprovado pelo Comitê de Ética em Pesquisa do Centro Universitário do Planalto de Araxá (UNIARAXÁ - 00397/04). Cada participante foi informado sobre a pesquisa (Carta de Informação) e assinou um Termo de Consentimento Livre e Esclarecido.

\section{RESULTADOS}

As médias globais de Burnout da amostra foram: 20,2\%, 6,8\% e 34,7\% para exaustão emocional, despersonalização e diminuição da realização pessoal, respectivamente. Esses percentuais são classificados como níveis médios de Burnout em todas as dimensões (Figura 1).

Figura 1 - Médias de Burnout na amostra nas diferentes dimensões da síndrome

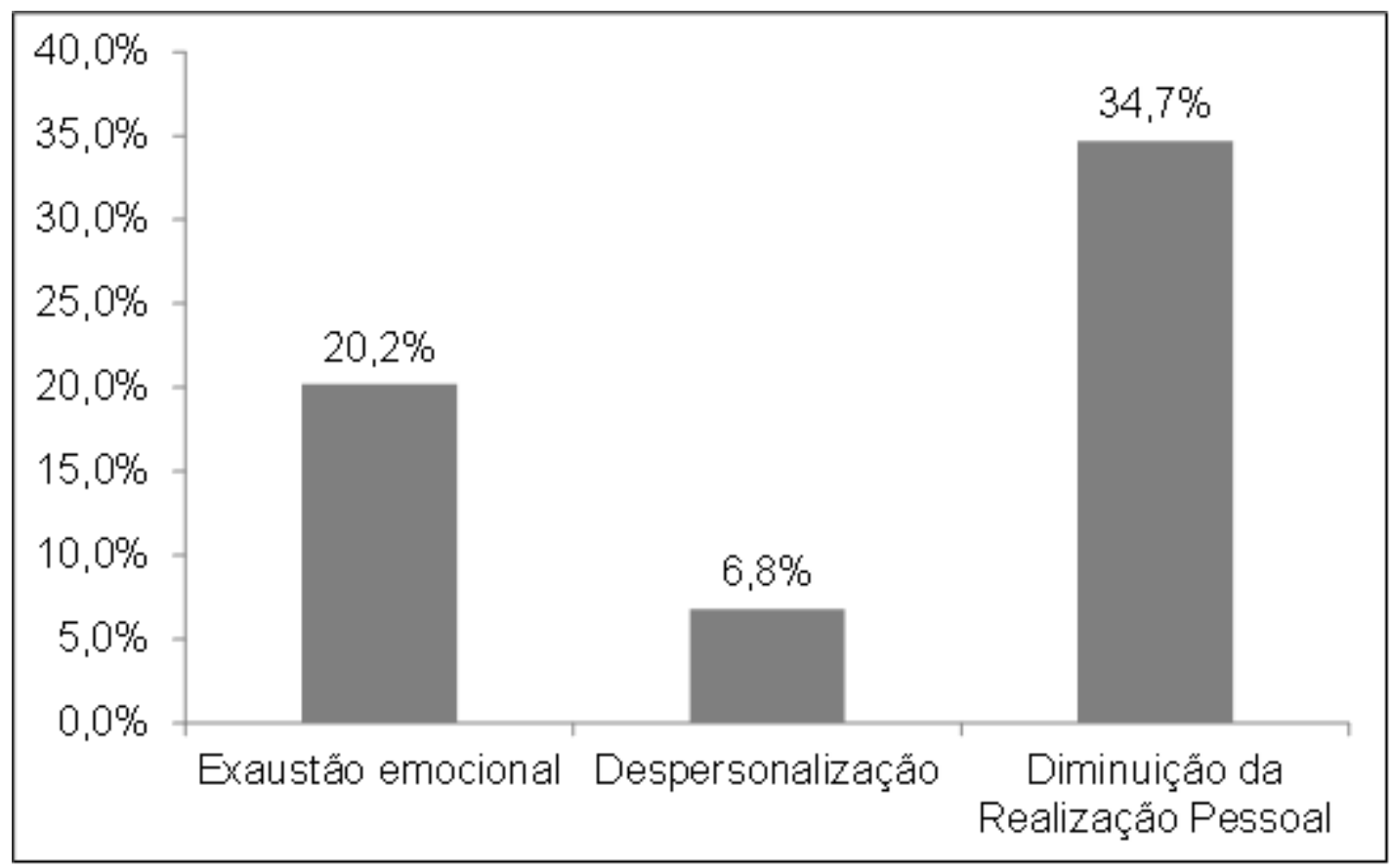

Fonte: Dados da pesquisa.

A Figura 2 refere-se à análise individual. Observou-se um nível alto nas três dimensões simultaneamente em $6,7 \%$ (5 profissionais) da amostra. A presença de pelo menos uma dimensão em nível alto foi encontrada em $62,5 \%$ (47 profissionais). 
Figura 2 - Distribuição dos voluntários quanto à presença de Burnout em nível alto

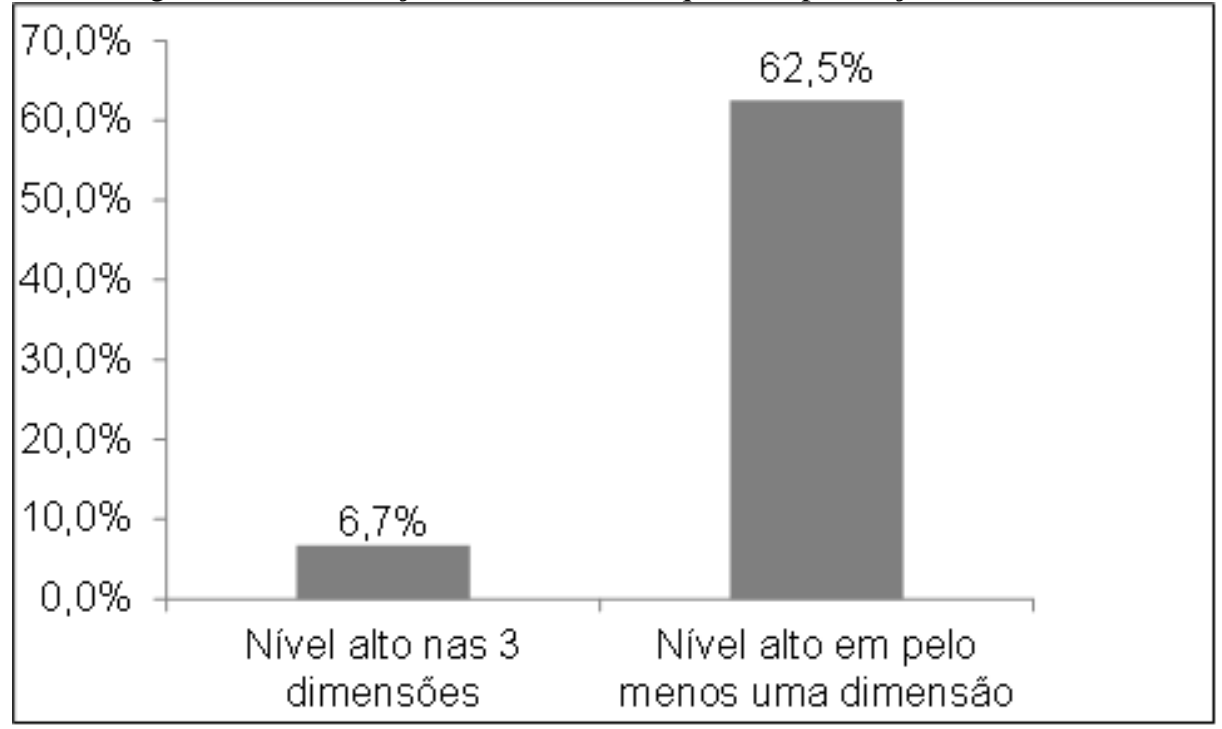

Fonte: Dados da pesquisa.

A distribuição percentual dos participantes quanto ao Burnout em suas diferentes dimensões e níveis está na Figura 3. Tendo em vista que uma pessoa em nível médio já possa apresentar algum tipo de sofrimento, é interessante somar os níveis médio e alto, desta forma tem-se para exaustão emocional, despersonalização e diminuição da realização pessoal os índices de 54,5\%, 46,6\% e 60,0\%, respectivamente.

Figura 3 - Distribuição dos participantes quanto ao Burnout em nível alto, médio e baixo em suas diferentes dimensões

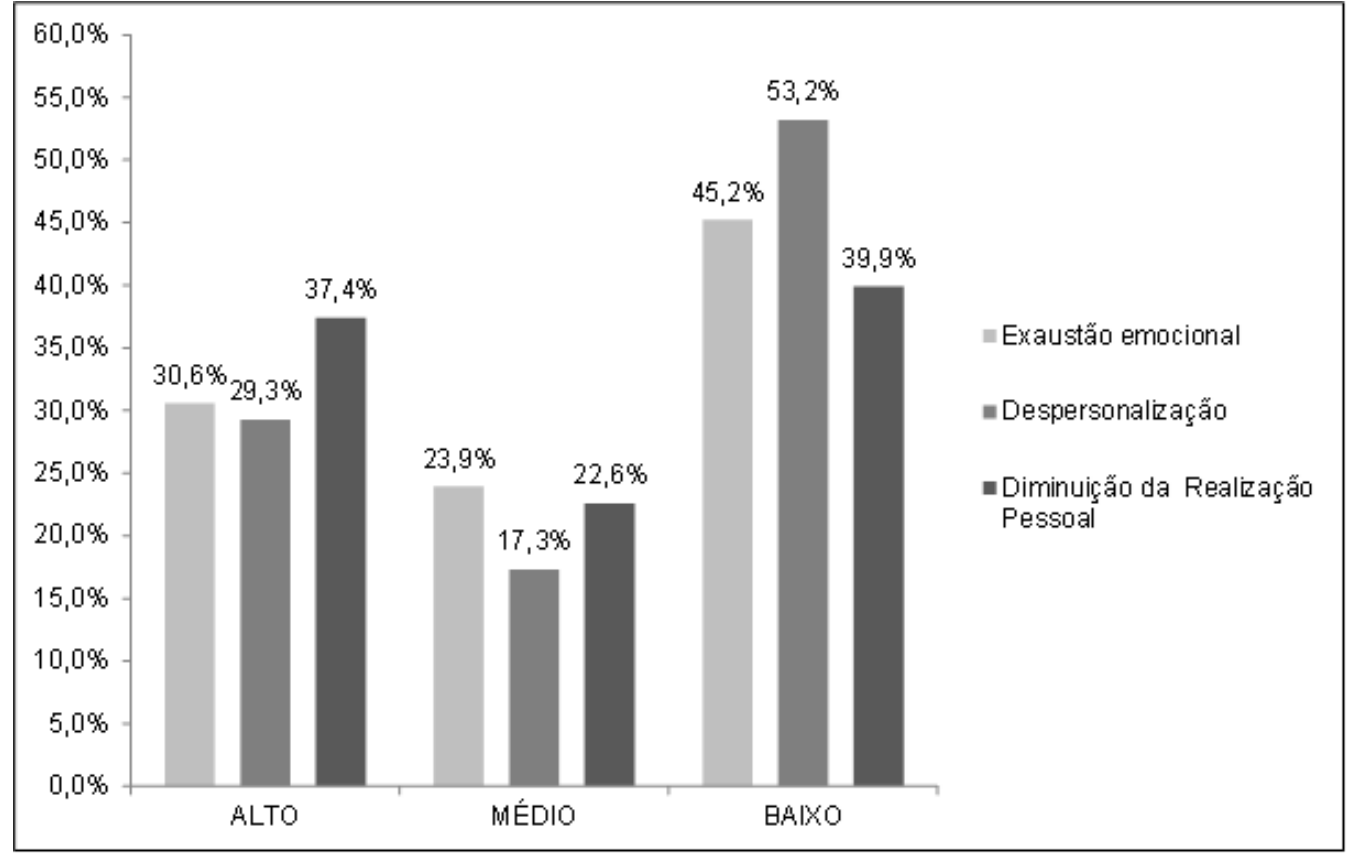

Fonte: Dados da pesquisa. 


\section{DISCUSSÃO}

Sabe-se que o Burnout se relaciona principalmente a fatores organizacionais e ocorre quando o profissional se depara com frustrações ou sobrecarga de trabalho e aumenta seus esforços para cumprir esses desafios. Assim, ele compensa o sofrimento psicológico com esse esforço extra (GISBERT; FAYOS, 2008).

Fatores externos e internos, bem como psicológicos e comportamentais, incidem diretamente no desenvolvimento do Burnout. Uma jornada excessiva de trabalho, indisciplina, falta de autonomia, perfeccionismo, autoestima baixa, negativismo, escolha profissional equivocada, falta de preparo e competência são exemplos de fatores envolvidos na origem e agravamento do quadro (ABREU et al., 2015).

Além disso, as más condições para o atendimento aos pacientes, os baixos salários, a carga horária elevada e um ambiente de trabalho desfavorável favorecem o surgimento de Burnout nos profissionais de saúde, o que interfere inclusive na relação terapêutica do profissional com seu paciente (AIKEN et al., 2002).

O presente estudo reforça a importância do estresse ocupacional, que deve ser fonte de preocupação e reconhecimento como um sério comprometedor do bem-estar dos indivíduos. Observou-se a presença de Burnout na amostra, detectando-se que mais da metade dela apresenta pelo menos uma dimensão do Burnout em nível alto.

Segundo Tucunduva et al. (2006), por não haver consenso na literatura para interpretação do questionário Maslach, e, consequentemente, o diagnóstico de Burnout, os resultados devem ser analisados de diferentes maneiras. É importante avaliar a presença de níveis altos em todas as dimensões, mas também determinar os que estão em risco de desenvolvimento.

Ramirez et al. (1996) definem o Burnout quando se tem níveis altos nas três dimensões. Já para Grunfeld et al. (2000) o diagnóstico de Burnout é quando o indivíduo apresenta nível alto em pelo menos uma das dimensões.

Segundo o critério de Ramirez et al. (1996), o diagnóstico de Burnout no presente estudo foi obtido em 6,7\% e pelos critérios de Grunfeld et al. (2000) em 62,5\%. Esses achados foram maiores que os obtidos em estudo realizado com 151 profissionais de enfermagem de um hospital de Tubarão, Santa Catarina (MOREIRA et al. 2009), que pelo critério de Ramirez et al. (1996) não houve diagnóstico da síndrome e pelo de Grunfeld et al. (2000) 35,7\% dos entrevistados apresentaram Burnout.

De forma similar, o estudo de Silva et al. (2015) realizado com trabalhadores de enfermagem intensivistas, no Rio de Janeiro (RJ), encontrou a prevalência de 55,3\% segundo critérios de Grunfeld et al. (2000).

No estudo realizado por Guido et al. (2012) com 37 residentes multiprofissionais da Universidade Federal de Santa Maria observou-se que 37,84\% apresentaram alta exaustão emocional; 43,24\%, alta despersonalização e $48,65 \%$, diminuição da realização pessoal. Em comparação aos resultados obtidos aqui, os índices foram muito similares para a dimensão exaustão emocional. Já para as demais foram um pouco menores.

Grau, Suner e Garcia (2005), em investigação realizada com trabalhadores da área da saúde na Espanha, verificaram alto nível de exaustão emocional, principalmente em médicos e profissionais de enfermagem; alto nível de despersonalização, sobretudo em médicos, e baixa realização profissional, em técnicos e médicos. 
Jodas e Haddad (2009), em uma pesquisa com 61 trabalhadores de enfermagem atuantes no Pronto-Socorro do Hospital Universitário Regional do Norte do Paraná (HURNP), investigaram os sinais e sintomas de Burnout nos participantes. Apenas $8,2 \%$ da amostra estavam com Burnout, mas aqueles que apresentaram predisposição para desenvolver esse problema representaram porcentagem significativa $(54,1 \%)$.

Silva e Alchieri (2014) realizaram uma pesquisa com 1.040 fisioterapeutas registrados nos conselhos profissionais de várias regiões brasileiras (Norte, Nordeste, Sul, Sudeste e Centro-Oeste), encontrando um índice de $49 \%$ (509) dos fisioterapeutas com tendência a desenvolver Burnout.

Considerando as controvérsias quanto ao diagnóstico estabelecido de Burnout, o encontro de uma dimensão em nível elevado, bem como níveis intermediários em todas as dimensões, pode não definir totalmente o diagnóstico, mas alerta para o risco de desenvolvimento, o que é muito relevante, pois pode ser o ponto de partida para uma intervenção precoce e redução de agravos.

Vale salientar que, segundo Gil-Monte (2008), são necessários a realização de entrevista clínica e o emprego de outros métodos de avaliação psicológica para confirmar o diagnóstico e descartar problemas que possam estar influenciando os sintomas avaliados. Só assim é possível uma análise ampla e que possa definir as consequências do Burnout e o grau de incapacidade para o exercício da atividade laboral.

\section{CONCLUSÃO}

De acordo com os resultados obtidos é possível concluir que há Burnout em alguns profissionais e outros estão em risco para a síndrome, o que pode comprometer a saúde e a qualidade de vida, bem como ter repercussões negativas no desempenho profissional desses trabalhadores.

\section{AGRADECIMENTOS}

Agradecemos à Fundação de Amparo à Pesquisa do estado de Minas Gerais - Fapemig.

\section{REFERÊNCIAS}

ABREU, S. A. et al. Determinação dos sinais e sintomas da síndrome de Burnout através dos profissionais da saúde da Santa Casa de Caridade de Alfenas Nossa Senhora do Perpétuo Socorro. Revista da Universidade Vale do Rio Verde, v. 13, n. 1, p. 204-238, 2015.

AIKEN, L. H.; et al. Hospital nurse staffing and patient mortality, nurse burnout, and job dissatisfaction. Jama, v. 288, p. 1.987-1.993, 2002.

ARANDA-BELTRÁN C. et al. Factores psicosociales laborales y síndrome de burnout en médicos del primer nivel de atención. Investigación en Salud, v. 6, n. 1, 2004.

BENEVIDES-PEREIRA, A. M. T. Burnout: quando o trabalho ameaça o bem-estar do trabalhador. 3. ed. São Paulo: Casa do Psicólogo, 2008.

BENEVIDES-PEREIRA, A. M. T. MBI - Maslach Burnout Inventory e suas adaptações para o Brasil [resumo]. In: REUNIÃO ANUAL DE PSICOLOGIA, 32., 2001, Rio de Janeiro. Anais... Rio de Janeiro. 2001.

CAMPOS, R. G. Burnout: uma revisão integrativa na enfermagem oncológica. 2005. 150 f. Dissertação (Mestrado) - Escola de Enfermagem de Ribeirão Preto, USP, São Paulo, 2005. 150 f. 
CARVALHO, L.; MALAGRIS L. E. N. Avaliação do nível de stress em profissionais de saúde. Estudos e Pesquisas em Psicologia, v. 7, p. 570-582, 2007.

DIAS, S.; QUEIROS, C.; CARLOTTO, M. S. Síndrome de Burnout e fatores associados em profissionais da área da saúde: um estudo comparativo entre Brasil e Portugal. Aletheia [on-line], v. 32, p. 4-21, 2010.

FRANÇA, T. L. B. et al. Síndrome de Burnout: características, diagnóstico, fatores de risco e prevenção. Revista de Enfermagem Ufpe, on-line, v. 8, n. 10, p. 3.539-3.546, 2014.

GIL-MONTE, P. R. Evaluación psicométrica del síndrome de quemarse por el trabajo (burnout): el cuestionario "CESQT". In GARRIDO J. (Org.). Power's Management. Barcelona: Planeta, 2008.

GISBERT, M. F. S.; FAYOS, E. J. G. Montesinos MDH. Burnout en fisioterapeutas españoles. Psicothema, v. 20, n. 3, p. 361-368, 2008.

GRAU, A.; SUNER, R.; GARCIA, M. M. Desgaste profesional en el personal sanitario y su relación con los factores personales y ambientales. Gaceta Sanitária, v. 19, n. 6, p. 463-470, 2005.

GRUNFELD, E. et al. Cancer care workers in Ontario: prevalence of burnout, job stress and job satisfaction. CMAJ, v. 163, p. 166$169,2000$.

GUIDO, L. A. et al. Síndrome de Burnout em residentes multiprofissionais de uma universidade pública. Revista da Escola de Enfermagem da USP, v. 46, n. 6, p. 1.477-1.483, 2012.

JODAS, D. A.; HADDAD, M. C. L. Síndrome de Burnout em trabalhadores de enfermagem de um pronto-socorro de hospital universitário. Acta Paulista de Enfermagem, v. 22, n. 2, p. 192-197, 2009.

MASLACH, C.; SCHAUFELI, W. B.; LEITER, M. P. Job Burnout. The Annual Review of Psychology, v. 52, p. 397-442, 2001.

MOREIRA, D. S. et al. Prevalência da síndrome de burnout em trabalhadores de enfermagem de um hospital de grande porte da Região Sul do Brasil. Cadernos de Saúde Pública, v. 25, n. 7, p. 1.559-1.568, 2009.

MULATO, S. C. O docente universitário em enfermagem e a Síndrome de Burnout: uma questão de educação para a saúde. 2008. 149 f. Dissertação (Mestrado) - Escola de Enfermagem de Ribeirão Preto, USP, São Paulo, 2008.

RAMIREZ, A. J. et al. W. M. Mental health of hospital consultants: the effects of stress and satisfaction at work. Lancet, v. 347, p. 724-728, 1996.

RIOS, I. C. Humanização e ambiente de trabalho na visão de profissionais da saúde. Saúde e Sociedade, v. 17, n. 4, p. 151-160, 2008.

ROSSI, A. M.; PERREWÉ, P. L.; SAUTER, S. L. Stress e qualidade de vida no trabalho: perspectivas atuais da saúde ocupacional. São Paulo: Atlas, 2005.

SANTOS, A. M. S. R. S. Burnout nos enfermeiros dos serviços de psiquiatria de doentes agudos - um contributo para a gestão. Dissertação (Mestrado em Gestão dos Serviços de Saúde). Lisboa: ISCTE. Business School Instituto Superior de Ciências do Trabalho e da Empresa, 2009.

SILVA, T. L. A.; ALCHIERI, J. C. Socioeconomic and demographic aspects related to stress and the burnout syndrome among Brazilian physiotherapists. Salud Ment, v. 37, n. 3, p. 233-238, 2014.

SILVA, J. L. L. et al. Psychosocial factors and prevalence of burnout síndrome among nursing workers in intensive care units. Revista Brasileira de Terapia Intensiva, v. 27, n. 2, p. 125-133, 2015. 
TUCUNDUVA, L. T. C. M. et al. A síndrome da estafa profissional em médicos cancerologistas brasileiros. Revista da Associação Médica Brasileira, v. 52, n. 2, p. 108-112, 2006.

XAVIER, J. W. O.; RIOS, O. F. L.; FRANÇA-BOTELHO, A. C. Qualidade de vida no trabalho, o desafio de vencer a síndrome de Burnout e suas consequências. Saúde e Pesquisa, v. 6, n. 1, p. 117-121, 2013. 